Rev. Biol. Neotrop. 10(2): 18-25. 2013

\title{
OCIABILIDADE DE ESPÉCIES DA MATA ATLÂNTICA COM A EXótICA inVASORA ARTOCARPUS HETEROPHYLLUS LAM.
}

\author{
Juliano Ricardo Fabricante \\ Universidade Federal do Vale do São Francisco (UNIVASF), Centro de Referência para Recupe- \\ ração de Áreas Degradadas (CRAD), BR 407, Km 12, Lote 543, s/n, Projeto de Irrigação Nilo \\ Coelho - C1, Petrolina, PE, Brasil - CEP: 56.300-000. E-mail: julianofabricante@hotmail.com
}

Resumo: O objetivo do presente estudo foi de avaliar a sociabilidade de espécies da Mata Atlântica nordestina com a exótica invasora $A$. heterophyllus, buscando nortear programas de controle e recuperação de áreas invadidas pelo táxon. Em 10 parcelas de $100 \mathrm{~m}^{2}$ que continham em seus centros geométricos um espécime adulto de $A$. heterophyllus, todos os indivíduos arbustivo-arbóreos foram contabilizados. Por meio do índice de associação (IA) e do coeficiente de correlação de Pearson $(\rho)$ foi avaliado o desempenho das espécies nativas (dado pela média aritmética entre o IA e $\rho$ ). A média de indivíduos de $A$. heterophyllus por parcela foi de $1.195,5 \pm 846,8$. Foram encontradas 44 espécies e duas morfoespécies associadas à exótica invasora, das quais, 19 apresentaram mais de cinco indivíduos e foram categorizadas quanto aos seus desempenhos. Do rol de espécies associadas a $A$. heterophyllus, muitas apresentam atributos ecológicos que permite a substituição da exótica invasora.

Palavras-chave: conservação da biodiversidade, Hotspots, invasão biológica,jaca.

Abstract: Sociability of Atlantic Forest species with the alien Artocarpus heterophyllus Lam. - The aim of this study was to evaluate the sociability of the northeastern Atlantic Forest species with invasive exotic $A$. heterophyllus, seeking to guide control programs and recovery areas invaded by taxon. In 10 plots of $100 \mathrm{~m}^{2}$ containing in their geometric centers one adult specimen of $A$. heterophyllus, all tree species were recorded. By means of the index of association (IA) and the Pearson correlation coefficient $(\rho)$ evaluated the performance of native species (given by the arithmetic mean between the IA the $\rho$ ). The average number of individuals of $A$. heterophyllus per share was $1,195.5 \pm 846.8$. 44 species were found and two morphospecies associated with invasive exotic, of which 19 had more than five individuals and were categorized as to their performance. The list of species associated with $A$. heterophyllus, many have ecological attributes that allow the replacement of invasive exotic.

KeY wORDs: biodiversity conservation, biological invasion, Hotspots, jackfruit.

\section{INTRODUÇÃo}

A pelas invasões biológicas sobre a economia e os ambientes naturais (Pimentel et al., 2001; Rejmánek et al., 2005; Reaser et al., 2007; Lawes \& Grice, 2007; Andrade et al., 2009, 2010; SMA, 2010; Zenni \& Ziller, 2011), a erradicação de exóticas invasoras de importância forrageira para a fauna nativa ainda geram discussões. A jaqueira (Artocarpus heterophyllus Lam.), árvore originária do sudeste asiático (Chaves et al., 1967), introduzida no Brasil por causa de seus frutos carnosos, é um desses casos. Ao mesmo tempo em que é fonte de alimento para animais (Haq, 2006; Novelli et al., 2010), A. heterophyllus representa uma ameaça significativa a fitodiversidade da Mata Atlântica (Boni et al., 2009; Abreu \& Rodrigues, 2010; Araújo et al., 2012; Fabricante et al., 2012), que possui em seus domínios mais de 20.000 espécies, das quais $40 \%$ endêmicas (Conservation International, 2012).

O conhecimento da flora nativa associada a espécies como $A$. heterophyllus, pode fornecer informações substanciais para programas de controle e recuperação de áreas invadidas. Por meio de ferramentas matemáticas simples, é possível estabelecer um rol de táxons zoocóricos capacitados a coabitar com as exóticas invasoras. 
Nesse sentido, a similaridade de Jaccard (Müller-Dombois \& Ellemberg, 1974), comumente utilizada para avaliar a parecença florística entre parcelas ou áreas, pode ser empregada para ponderar sobre a associação entre espécies (Drumond et al., 1982). Ainda, o coeficiente de correlação linear de Pearson, que visa avaliar a interdependência entre duas variáveis (Rodgers \& Nicewander, 1988), pode determinar como a abundância das espécies varia uma em razão da outra.

Teoricamente as espécies com maior desempenho nessas análises, serão aquelas que apresentam certa tolerância à presença e aos efeitos causados pelas exóticas invasoras no meio biofísico. Em particular para $A$. heterophyllus, já foi constatado que a mesma possui substâncias alelopáticas (Perdomo \& Magalhães, 2007), aumenta a abundância de indivíduos por área e altera a química e a fertilidade dos solos (Fabricante et al., 2012).

Assim, o objetivo do presente estudo foi avaliar a sociabilidade de espécies da Mata Atlântica nordestina com a exótica invasora $A$. heterophyllus, buscando nortear programas de controle e recuperação de áreas invadidas pelo táxon.

\section{Material e Métodos}

O local de estudo caracteriza-se por uma Área de Preservação Permanente (APP) de Mata Atlântica (Brejo de Altitude) do Campus de Ciências Agrárias (CCA), Universidade Federal da Paraíba (UFPB), município de Areia, PB. O fragmento possui pouco mais de 50 ha $\left(6^{\circ} 58^{\prime} 1,9^{\prime \prime} \mathrm{S}\right.$ e $35^{\circ} 43^{\prime} 4,2^{\prime \prime}$ W; $592 \mathrm{~m}$ de altitude média) e está situado em uma região sob o clima do tipo $\mathrm{As}^{\prime}$ (classificação de Koppen), com precipitação média anual superior a $1.400 \mathrm{~mm}$. Os solos da região são predominantemente de Argissolos (Embrapa, 2006).

Segundo estudo desenvolvido nesse fragmento, $A$. heterophyllus possui uma densidade de 2.391 indivíduos/ha, cujos espécimes se distribuem principalmente em suas bordas, contudo, não restritos a elas (Fabricante et al., 2012). Outras informações sobre a área de estudo, assim como sobre os impactos causados pela espécie nos sítios invadidos, podem ser obtidos no trabalho supramencionado.

Em 10 parcelas com dimensão de $10 \times 10$ $\mathrm{m}\left(100 \mathrm{~m}^{2}\right)$ que havia em seus centros geométricos um indivíduo adulto de $A$. heterophyllus, todos os espécimes da exótica invasora e do componente arbustivo-arbóreo autóctone foram contabilizados.

Para avaliar a associação das espécies nativas com $A$. heterophyllus, foi utilizado o coeficiente binário de Jaccard, conforme descrito por Drumond et al. (1982) (Tabela 1). Já para inferir sobre a correlação entre os táxons, foi empregado o coeficiente de Pearson $(\rho)$ (Rodgers \& Nicewander, 1988), cujas forças de correlação foram consideradas segundo Levin (1987) (Tabela 2). Só foram analisadas as espécies que apresentaram no mínimo cinco indivíduos.
Tabela 1 - Índice de associação (IA) e respectivas forças (segundo Drumond et al. 1982).

\begin{tabular}{lc}
\hline Força do IA & IA \\
\hline Muito Alta & $1-0,8$ \\
Alta & $0,79-0,6$ \\
Média & $0,59-0,4$ \\
Baixa & $0,39-0,2$ \\
Muito Baixa & Menor que 0,19 \\
\hline
\end{tabular}

Tabela 2 - Coeficiente de Pearson $(\rho)$ e respectivas forças (adaptado de Levin 1987).

\begin{tabular}{lc}
\hline Força do $\boldsymbol{\rho}$ & $\boldsymbol{\rho}$ \\
\hline Positiva Perfeita & 1 \\
Positiva Muito Forte & $0,70-0,99$ \\
Positiva Substancial & $0,50-0,69$ \\
Positiva Moderada & $0,30-0,49$ \\
Positiva Baixa & $0,10-0,29$ \\
Positiva Ínfima & $0,01-0,09$ \\
Nula & 0 \\
Negativa Ínfima & $-0,01--0,09$ \\
Negativa Baixa & $-0,10--0,29$ \\
Negativa Moderada & $-0,30--0,49$ \\
Negativa Substancial & $-0,50--0,69$ \\
Negativa Muito Forte & $-0,70--0,99$ \\
Negativa Perfeita & -1 \\
\hline
\end{tabular}

Visando categorizar o desempenho das espécies nativas quanto aos resultados conjuntos do índice de associação (IA) e coeficiente de Pearson $(\rho)$, foi feita a média aritmética entre os mesmos. As categorias de desempenho foram dadas conforme segue na Tabela 3.

Tabela 3 - Média aritmética entre o índice de associação (IA) e o coeficiente de Pearson $(\rho)$ e respectivas categorias de desempenho.

\begin{tabular}{lc}
\hline Desempenho & $\frac{\text { IA }+\rho}{2}$ \\
\hline Excelente & $1-0,8$ \\
Muito Bom & $0,79-0,6$ \\
Bom & $0,59-0,4$ \\
Médio & $0,39-0,2$ \\
Ruim & $0,19-0$ \\
Muito Ruim & Valores Negativos \\
\hline
\end{tabular}


Para ponderar sobre as potencialidades das espécies com maior desempenho nas avaliações, executou-se uma ampla revisão bibliográfica nas principais bases de dados científicos nacionais e internacionais. A classificação taxonômica das espécies foi elaborada de acordo com o Sistema APG III (2009). A síndrome de dispersão das espécies baseou-se em literatura específica (Silva \& Rodal, 2009; Marangon et al., 2010; Silva et al., 2012). As análises estatísticas foram executadas utilizando-se o software Past $2.17 b^{\odot}$ (Hammer et al., 2001).

\section{Resultados e discussão}

A média de indivíduos de $A$. heterophyllus por parcela foi de $1.195,5 \pm 846,8$ (média \pm desvio padrão). Foram encontradas 44 espécies e duas morfoespécies associadas à exótica invasora, das quais, 19 supriram o critério de inclusão estabelecido, ou seja, apresentaram mais de cinco indivíduos (Tabela 4).

Tabela 4 - Número de indivíduos (n), índice de associação (IA), coeficiente de Pearson ( $\rho$ ), média aritmética entre o "IA" e o " $\rho$ " e síndrome de dispersão de espécies de Mata Atlântica nordestina, associadas à exótica invasora Artocarpus heterophyllus, Areia, PB.

\begin{tabular}{|c|c|c|c|c|c|c|c|c|}
\hline Espécies & $\mathbf{n}$ & IA & $\begin{array}{l}\text { Força } \\
\text { do IA }\end{array}$ & $\boldsymbol{\rho}$ & Força do $p$ & $\frac{\mathrm{IA}+\rho}{2}$ & Desempenho & $\begin{array}{c}\text { Síndrome de } \\
\text { Dispersão }\end{array}$ \\
\hline Sorocea bonplandii & 13 & 0,4 & Média & 0,839 & Positiva Muito Forte & 0,620 & Muito Bom & Zoocórica \\
\hline Guapira opposita & 13 & 0,6 & Alta & 0,493 & Positiva Moderada & 0,547 & Bom & Zoocórica \\
\hline Cupania sp. & 10 & 0,4 & Média & 0,420 & Positiva Moderada & 0,410 & Bom & - \\
\hline Erythroxylum simonis & 31 & 0,8 & Muito Alta & $-0,007$ & Negativa Ínfima & 0,397 & Médio & Zoocórica \\
\hline Inga ingoides & 11 & 0,7 & Alta & $-0,060$ & Negativa Ínfima & 0,320 & Médio & Zoocórica \\
\hline Piper aduncun & 13 & 0,4 & Média & 0,183 & Positiva Baixa & 0,292 & Médio & Zoocórica \\
\hline Albizia polycephala & 20 & 0,6 & Alta & $-0,039$ & Negativa Ínfima & 0,281 & Médio & Autocórica \\
\hline Eschweilera ovata & 7 & 0,6 & Alta & $-0,063$ & Negativa Ínfima & 0,269 & Médio & Autocórica \\
\hline Schefflera morototoni & 6 & 0,4 & Média & 0,088 & Positiva Ínfima & 0,244 & Médio & Zoocórica \\
\hline Brosimum guianense & 29 & 0,5 & Média & $-0,032$ & Negativa Ínfima & 0,234 & Médio & Zoocórica \\
\hline Allophylus laevigatus & 9 & 0,3 & Baixa & 0,101 & Positiva Baixa & 0,201 & Médio & Zoocórica \\
\hline Myrcia silvatica & 13 & 0,4 & Média & $-0,069$ & Negativa Ínfima & 0,166 & Ruim & Zoocórica \\
\hline Thyrsodium spruceanum & 89 & 0,5 & Média & $-0,170$ & Negativa Baixa & 0,165 & Ruim & Zoocórica \\
\hline Talisia esculenta & 9 & 0,4 & Média & $-0,182$ & Negativa Baixa & 0,109 & Ruim & Zoocórica \\
\hline Himatanthus phagedaenicus & 9 & 0,2 & Baixa & $-0,088$ & Negativa Ínfima & 0,056 & Ruim & Anemocórica \\
\hline Myrcia sp1. & 5 & 0,3 & Baixa & $-0,200$ & Negativa Baixa & 0,050 & Ruim & - \\
\hline Cupania revoluta & 17 & 0,5 & Média & $-0,477$ & Negativa Moderada & 0,012 & Ruim & Zoocórica \\
\hline Machaerium aculeata & 7 & 0,4 & Média & $-0,420$ & Negativa Moderada & $-0,010$ & Muito Ruim & Anemocórica \\
\hline
\end{tabular}

Apenas uma espécie $(5,3 \%)$ apresentou força de associação "muito alta" com A. heterophyllus. As demais, cinco $(26,3 \%)$ foram consideradas "altas", dez (52,6\%), "médias" e quatro $(21,1 \%)$, "baixas". Nenhuma das espécies avaliadas estava em apenas uma parcela, não havendo desta forma, associações com força "muito baixa".

A maioria das espécies apresentou correlação "negativa" com $A$. heterophyllus (68,4\%). Dentre as com correlação "positiva" $(31,6 \%)$, uma $(5,3 \%)$ foi "muito forte", duas $(10,5 \%)$, "moderada" e "baixa" e uma (5,3\%) "ínfima".

Mais da metade $(57,9 \%)$ das espécies avaliadas apresentaram desempenho satisfatório. Uma $(5,3 \%)$ apresentou desempenho "muito bom", duas $(10,5 \%)$, "bom" e oito $(42,1 \%)$ médio. Do restante das espécies, seis $(31,6 \%)$ obtiveram desempenho "ruim" e duas, (10,5\%) "muito ruim".
A espécie com melhor desempenho foi Sorocea bonplandii ("muito bom"). A mesma apresentou 13 indivíduos, distribuídos em quatro parcelas (IA $=0,4)$, e correlação "positiva muito forte" com A. heterophyllus. Além desses resultados, S. bonplandii destaca-se por possuir ampla distribuição geográfica, podendo ser encontrada no Brasil, Argentina e Paraguai (García et al., 2011). No País, ela ocorre nos domínios do Cerrado (Oliveira \& Felifili, 2005; Ribeiro \& Felfili, 2009) e da Mata Atlântica (Ferraz et al., 2004; Neri et al., 2007), biomas em que $A$. heterophyllus apresenta maior susceptibilidade a invasão (Fabricante et al., 2012).

Outros aspectos ecológicos de $S$. bonplandii a torna ainda mais promissora para os objetivos deste estudo. $O$ táxon produz frutos muito apreciados por macacos (Aguiar et al., 2003; Ludwig et al., 2005) e aves (Mikich, 2002), apresenta alta 
sociabilidade com outras plantas nativas (Lindenmaier \& Budke, 2006; Suhs \& Budke, 2011) e é auto regenerante em áreas com diferentes condições de conservação e manejo (Sartori et al., 2002; Scipioni et al., 2009; Ribeiro et al., 2010).

O segundo táxon com melhor desempenho ("bom") foi Guapira opposita, que apresentou 13 indivíduos, distribuídos em seis unidades amostrais ( $\mathrm{IA}=0,6)$, e correlação "positiva moderada" com a exótica invasora. Além de possuir alguns atributos semelhantes à espécie anterior, e.g. : ampla distribuição (Sá 2012), ocorrência no Cerrado (Felfili et al., 2002; Pereira-Silva et al., 2004) e na Mata Atlântica (Andrade et al., 2006; Liebsch et al., 2007; Marchioretto et al., 2012), e fonte de alimento para macacos (Koch, 2008) e aves (Fadini \& Marco Jr., 2004; Scherer et al., 2007), G. opposita ainda é considerada um importante hospedeiro (forófito) para as epífitas da Mata Atlântica (Kersten \& Silva, 2001; Bonnet at al., 2007), componente este, inexistente sobre A. heterophyllus na área de estudo e em outros fragmentos da Região (obs. pess.).

A espécie apresenta grande plasticidade ambiental, sendo heliófita a ciófita (Lorenzi, 2002) e contribui para o acúmulo significativo de serapilheira (Pires et al., 2006), elemento notoriamente importante para a integridade dos solos e produtividade das florestas (Pritcehtt, 1979). G. opposita também se destaca por ser uma espécie muito procurada por insetos galhadores (Bregonci et al., 2010; Maia \& Oliveira, 2010), contribuindo assim, para a manutenção da diversidade da entomofauna (Maia, 2001).

Apesar do desempenho menor, outras espécies zoocóricas apresentaram alguns resultados e possuem características que devem ser levadas em consideração. Erythroxylum simonis, por exemplo, obteve o segundo maior número de indivíduos (31) e o maior índice de associação (0,8 - "muito alta"). Endêmica do Nordeste brasileiro (Loiola, 2012) e frutificando em dois períodos distintos no ano (Loiola et al., 2007), a utilização desta espécie tem dupla função: preservar os recursos genéticos endêmicos da Região e garantir fonte de alimento para a fauna em diferentes momentos.

Uma das espécies mais comuns do estrato arbóreo adulto (Andrade et al., 2006; Brandão et al., 2009) e regenerante (Silva et al., 2007; Aparício et al., 2011) da Mata Atlântica nordestina, Thyrsodium spruceanum, foi a espécie mais abundante (89 ind.) associada a $A$. heterophyllus. No Brasil ela ocorre em Minas Gerais, Rio de Janeiro, Espírito Santo e nas Regiões Nordeste e Norte (Silva-Luz \& Pirani, 2012), cujo padrão de distribuição é considerado do tipo Amazônico-Atlântico (Rodal et al., 2005).

Por sua vez, Brosimum guianense foi a terceira espécie em número de indivíduos (29). Assim como S. bonplandii e $G$. opposita, B. guianense também se distribui pelo Cerrado e Mata Atlântica (Romaniuc-Neto et al., 2012). Além da ampla dispersão, seus frutos são consumidos por várias espécies de macacos (Stevenson et al., 2002; Port-Carvalho \& Ferrari, 2004; Pinto \& Setz, 2004), aves (Erard et al., 1989) e cervídeos (Gayot et al., 2004).

Do rol de espécies associadas a $A$. heterophyllus, muitas apresentam atributos ecológicos semelhantes às descritas até aqui. A maioria absoluta delas são zoocóricas (13 espécies - 68\%). Entre as outras seis, duas (10,5\%) são autocóricas, duas $(10,5 \%)$ anemocóricas e duas $(10,5 \%)$ não puderam ser determinadas, contudo, as características morfológicas dos frutos sugerem que ambas sejam dispersas por animais: Cupania sp. apresenta sementes com arilo e Myrcia sp., frutos carnosos.

Outra questão relevante é que existem representantes de distintos estratos da vegetação dentre os táxons amostrados, o que permite reproduzir as condições naturais das florestas (estratificação), e.g. Guapira opposita, Inga ingoides, Schefflera morototoni, Sorocea bonplandii, Thyrsodium spruceanum são árvores que compõe o dossel da mata; Brosimum guianense, Erythroxylum simonis e Piper aduncun, são arvoretas ou arbustos de sub-bosque dessas formações.

\section{Conclusões}

Os resultados deste estudo apontam a existência de várias espécies sociáveis a $A$. heterophyllus, que possuem atributos desejáveis para programas de controle e recuperação de áreas invadidas pela exótica invasora.

Sugere-se que a execução das ações de controle e recuperação de áreas invadidas por $A$. heterophyllus seja feita em três etapas:

(i) remoção dos regenerantes da exótica invasora e plantio consorciado de espécies autóctones zoocóricas - esse procedimento busca evitar a competição entre os indivíduos jovens de $A$. heterophyllus e as nativas;

(ii) eliminação contínua da regeneração natural de $A$. heterophyllus;

(iii) anelamento dos espécimes adultos da exótica invasora no momento em que as nativas atingirem a fase reprodutiva - esse procedimento busca eliminar completamente a exótica invasora somente quando as espécies autóctones forem capazes de dar suporte alimentar adequado a fauna nativa.

\section{ReferÊnCIAS}

Abreu, R.C.R. \& P.J.F.P. Rodrigues. 2010. Exotic tree Artocarpus heterophyllus (Moraceae) invades the Brazilian Atlantic Rainforest. Rodriguésia, 61: 677-688.

Aguiar, L.M., N.R. Reis, G. Ludwig \& V.J. Rocha. 2003. Dieta, área de vida, vocalizações e estimativas populacionais de Alouatta guariba (Humboldt, 1812) em um remanescente florestal do norte do estado do Paraná. Neotropica Primates, 11: 78-86. 
Andrade, L.A., J.R. Fabricante \& F.X. Oliveira. 2009. Invasão biológica por Prosopis juliflora (Sw.) DC.: impactos sobre a diversidade e a estrutura do componente arbustivo-arbóreo da caatinga no Estado do Rio Grande do Norte, Brasil. Acta Botanica Brasilica, 23:, 935-943.

Andrade, L.A., J.R. Fabricante \& F.X. Oliveira. 2010. Impactos da Invasão de Prosopis juliflora (Sw.) DC. (Fabaceae) sobre o Estrato Arbustivo-Arbóreo em Áreas de Caatinga no Estado da Paraíba, Brasil. Acta Scientiarum. Biological Sciencies, 32: 249-255.

Andrade, L.A., F.X. Oliveira, I.S. Nascimento, J.R. Fabricante, E.V.S.B. Sampaio \& M.R.V. Barbosa. 2006. Análise florística e estrutural de matas ciliares ocorrentes em Brejo de Altitude no Município de Areia, Paraíba. Revista Brasileira de Ciências Agrárias, 1: 31-40.

Aparício, W.C.S., L.C. Marangon, R.L.C. Ferreira, A.L.P. Feliciano, P.S. Aparicio \& R.F. Costa-Jr. 2011. Estrutura da regeneração natural de espécies arbóreas em um fragmento de Mata Atlântica, Pernambuco. Revista Brasileira de Ciências Agrárias, 6: 483-488.

APG. 2009. An update of the Angiosperm Phylogeny Group classification for the orders and families of flowering plants: APG III. Botanical Journal of the Linnean Society, 161: 105-121.

Araújo, K.C.T., J.R. FABRICANTE \& L.A. Andrade. 2012. Jaqueira: Uma Invasora na Mata Atlântica. Ciência Hoje, 49: 44-48.

Boni, R., F.Z. Novelli \& A.G. Silva. 2009. Um alerta para os riscos de bioinvasão de jaqueiras, Artocarpus heterophyllus Lam., na Reserva Biológica Paulo Fraga Rodrigues, antiga Reserva Biológica Duas Bocas, no Espírito Santo, Sudeste do Brasil. Natureza on line, 7: 51-55.

Bonnet, A., M.H. Queiroz, \& O.J. Lavoranti. 2007. Relações de bromélias epifíticas com características dos forófitos em diferentes estádios sucessionais da Floresta Ombrófila Densa, Santa Catarina, Brasil. Floresta, 37: 83-94.

Brandão, C.F.L.C., L.C. Marangon, R.L.C. Ferreira \& A.C.B. Lins-e-Silva. 2009. Estrutura fitossociológica e classificação sucessional do componente arbóreo em um fragmento de floresta atlântica em Igarassu - Pernambuco. Revista Brasileira de Ciências Agrárias, $4:$ 55-61.
Bregonci, J.M., P.V. Polycarpo \& V.C. Maia. 2010. Galhas de insetos do Parque Estadual Paulo César Vinha (Guarapari, ES, Brasil). Biota Neotropica, 10: 267-274.

Conservation International. 2012. Biodiversity Hotspots. Conservation International. <http:// www.biodiversityhotspots.org/Pages/default.aspx>. (Acesso em 12/12/2012).

Drumond, M.A., P.C.F. Lima, S.M. Souza \& J.L.S. Lima. 1982. Sociabilidade das espécies florestais da caatinga em Santa Maria da Boa Vista-PE. Boletim de Pesquisa Florestal, 4: 47-59.

EMBRAPA - Centro Nacional de Pesquisa de Solos. 2006. Sistema Brasileiro de Classificação de Solos. 2 ed. Rio de Janeiro: Embrapa Solos.

Erard, C., M.A. Théry \& D. Sabatier. 1989. Régime alimentaire de Rupicola rupicola (Cotingidae) en Guyane Française relations avec la frugivorie et la zoochorie. Terre Vie, 44: 47-74.

Fabricante, J.R., K.C.T. Araújo, L.A. Andrade \& J.V.A. Ferreira. 2012. Invasão Biológica de Artocarpus heterophyllus Lam. (Moraceae) em um Fragmento de Mata Atlântica no Nordeste do Brasil: Impactos sobre a Fitodiversidade e os Solos dos Sítios Invadidos. Acta Botanica Brasílica, 26: 399-407.

Fadini, R.F. \& P. Marco-Jr. 2004. Interações entre aves frugívoras e plantas em um fragmento de mata atlântica de Minas Gerais. Ararajuba, 12: 97-103.

Felfili, J.M., P.E. Nogueira, M.C. Silva-Jr., B.S. Marimon \& W.B.C. Delitti. 2002. Composição florística e fitossociológica do cerrado sentido restrito no município de Água Boa-MT. Acta Botanica Brasilica, 16: 103-112.

Ferraz, E.M.N., E.L. Araújo \& S.I. Silva. 2004. Floristic similarities between lowland and montane areas of Atlantic Coastal Forest in Northeastern Brazil. Plant Ecology, 174: 59-70.

García, M.G., L. Brítez, Y. González \& R.D. Arrúa. 2011. Morfoanatomia comparativa de Genipa americana L. (Rubiaceae) y Sorocea bonplandii (Baill.) W.C. Burger, Lanj. \& Wess. Boer (Moraceae) comercializadas en el mercado de San Lorenzo. Rojasiana, 10: 93-101.

Gayot, M., O. Henry, G. Dubost \& D. Sabatier. 2004. Comparative diet of the two forest cervids of the genus Mazama in French Guiana. Journal of Tropical Ecology, 20: 31-43. 
Hammer, O., D.A. Harper \& P.D. Ryan. 2001. PAST 2.17b. Palaeontolologia Eletronica, 4: 9.

Haq, N. 2006. Jackfruit, Artocarpus heterophylIus. Southampton Centre for Underutilised Crops, University of Southampton. 208p.

Kersten, R.A. \& S.M. Silva. 2001. Composição florística e estrutura do componente epifítico vascular em Floresta da planície litorânea na Ilha do Mel, Paraná, Brasil. Revista Brasileira de Botânica, 24: 213-226.

Koch, F. 2008. Dieta e comportamento de um grupo de Alouatta guariba clamitans Cabrera, 1940: uma relação de causa e efeito? Dissertação (Mestrado em Zoologia). Pontifícia Universidade Católica do Rio Grande Do Sul. 57p.

Lawes, R.A. \& A.C. Grice. 2007. Controlling infestations of Parkinsonia aculeata in a Riparian Zone at the landscape scale. Austral Ecology, 32: 287-293.

Levin, J. 1987. Estatística Aplicada às Ciências Humanas. 2 ed. Harper \& Row do Brasil. 392p.

Liebsch, D., R. Goldenberg \& M.C.M. Marques. 2007. Florística e estrutura de comunidades vegetais em uma cronoseqüência de Floresta Atlântica no Estado do Paraná, Brasil. Acta Botancia Brasilica, 21: 983-992.

Lindenmaier, D.S. \& J.C. Budke. 2006. Florística, diversidade e distribuição espacial das espécies arbóreas em uma Floresta Estacional na bacia do rio Jacuí, Sul do Brasil. Pesquisas, Botânica, 57: 193-216.

Loiola, M.I.B., M.F. Agra, G.S. Baracho \& R.T. Queiroz. 2007. Flora da Paraíba, Brasil: Erythroxylaceae Kunth. Acta Botanica Brasilica, 21: 473-487.

Loiola, M.I.B. 2012. Erythroxylaceae in Lista de Espécies da Flora do Brasil. Jardim Botânico do Rio de Janeiro. <http://floradobrasil. jbrj.gov.br/2012/FB007730>. (Acesso em 18/12/2012).

Lorenzi, H. 2002. Árvores Brasileiras - manual de identificação e cultivo de plantas arbóreas nativas do Brasil. 4 ed. Instituto Plantarum. $384 \mathrm{p}$.

Ludwig, G., L.M. Aguiar \& V.J. Rocha. 2005. Uma avaliação da dieta, da área de vida e das estimativas populacionais de Cebus nigritus (Goldfuss, 1809) em um fragmento florestal no Norte do Estado do Paraná. Neotropical Primates, 13:12-18.
Maia, V.C. 2001. The gall midges (Diptera, Cecidomyiidae) from three restingas of Rio de Janeiro State, Brazil. Revista Brasileira de Zoologia, 18: 583-629.

Maia, V.C. \& J.C. Oliveira. 2010. Galhas de insetos da Reserva Biológica Estadual da Praia do Sul (Ilha Grande, Angra dos Reis, RJ). Biota Neotropica, 10: 227-237.

Marangon, G.P., A.F. Cruz, W.B. Barbosa, G.H. Loureiro \& A.C. Holanda. 2010. Dispersão de sementes de uma comunidade arbórea em um remanescente de mata atlântica, Município de Bonito, PE. Revista Verde, 5: 80-87.

Marchioretto, M.S., A.P.U. Lippert \& M.G. Staudt. 2012. Padrões de distribuição geográfica da família Nyctaginaceae Juss. no Rio Grande do Sul. Pesquisas, Botânica, 63: 201-211.

Mikich, S.B. 2002. A dieta frugívora de Penelope superciliaris (Cracidae) em remanescentes de floresta estacional semidecidual no centro-oeste do Paraná, Brasil e sua relação com Euterpe edulis (Arecaceae). Ararajuba, 10: 207-217.

Muller-Dombois, D., \& H. Ellemberg. 1974. Aims and methods of vegetation ecology. John Wiley \& Sons. 547p.

Neri, A.C.A., C.S. Prado, G. Terra \& D.M.S. Matos. 2007. Consequência do uso e ocupação do solo sobre florestas ribeirinhas na região de Teresópolis, RJ. Revista Brasileira de Biociências, 5: 444-446.

Novelli, F.Z., R.P.G. Moreira, C. Duca \& A.G. Silva. 2010. O papel da barocoria na estruturação da população da jaqueira, Artocarpus heterophyllus Lam. na Reserva Biológica de Duas Bocas, Cariacica, Espírito Santo. Natureza on line, 8: 91-94.

Oliveira, E.C.L. \& J.M. Felfili. 2005. Estrutura e dinâmica da regeneração natural de uma mata de galeria no Distrito Federal, Brasil. Acta Botanica Brasilica, 19: 801-811.

Perdomo, M. \& L.M.S. Magalhães. 2007. Ação alelopática (Artocarpus heterophyllus) em laboratório. Floresta e Ambiente, 14: 52-55.

Pereira-Silva, E.F.L., J.E. Santos, P.Y. Kageyama \& E. Hardt. 2004. Florística e fitossociologia dos estratos arbustivo e arbóreo de um remanescente de cerradão em uma Unidade de Conservação do Estado de São Paulo. Revista Brasileira de Botânica, 27: 533-544. 
Pinto, L.P. \& E.Z.F. Setz. 2004. Diet of Alouatta belzebul discolor in an Amazonian Rain Forest of Northern Mato Grosso State, Brazil. International Journal of Primatology, 25: 1197-1211.

Pires, L.A., R.M. Britez, G. Martel, \& S.N. Pagano. 2006. Produção, acúmulo e decomposição da serapilheira em uma restinga da Ilha do Mel, Paranaguá, PR, Brasil. Acta Botanica Brasilica, 20: 173-184.

Pimentel, D., S. McNair, J. Janecka, J. Wightman, C. Simmonds, C. O'Connell, E. Wong, L. Russel, J. Zern, T. Aquino, \& T. Tsomondo. 2001. Economic and Environmental Threats of Alien Plant, Animal, and Microbe Invasions. Agriculture, Ecosystems and Environment, 84: 1-20.

Port-Carvalho, M. \& S.F. Ferrari. 2004. Occurrence and diet of the black bearded saki (Chiropotes satanas satanas) in the fragmented landscape of western Maranhão, Brazil. Neotropical Primates 12: 17-21.

Pritchett, W.L. 1979. Properties and management of forest soils. John Wiley and Sons. 500p.

Reaser, J.K., L.A. Meyerson, Q. Cronk, M. Poorter, L.G. Eldrege, E. Green, M. Kairo, P. Latasi, R.N. Mack, J. Mauremootoo, D. O'dowd, W. Orapa, S. Sastroutomo, A. Saunders, C. Shine, S. Thrainsson \& L. Vaiutu. 2007. Ecological and socioeconomic impacts of invasive alien species in island ecosystems. Environmental Conservation, 34: 98-111.

Rejmánek M., D.M. Richardson \& P. Pysek. 2005. Plant invasions and invasibility of plant communities. p. 332-355. In: VanDer-Maarel, E. (ed.), Vegetation ecology. Blackwell Publishers. 395p.

Ribeiro, G.H.P.M. \& M.J. Felfili. 2009. Regeneração natural em diferentes ambientes da Mata de Galeria do Capetinga na Fazenda Água Limpa. Revista Cerne, 15: 1-9.

Ribeiro, S.C., L.A.G. Jacovine, C.P.B Soares, S.V. Martins, A.M.B. Nardelli \& A.L. Souza. 2010. Quantificação de biomassa e estimativa de estoque de carbono em uma capoeira da Zona da Mata mineira. Revista Árvore, 34: 495-504.

Rodal, M.J.N., M.F. Sales, M.J. Silva \& A.G. Silva. 2005. Flora de um Brejo de Altitude na escarpa oriental do planalto da Borborema, PE, Brasil. Acta Botanica Brasilica 19: 843-858.
Rodgers, J.L. \& W.A. Nicewander. 1988. Thirteen ways to look at the correlation coefficient. The American Statistician, 42: 59-66.

Romaniuc-Neto, S., J.P.P. Carauta, M.D.M. Vianna-Filho, R.A.S. Pereira, J.E.L.S. Ribeiro, A.F.P. Machado, A. Santos, G. Pelissari \& L.C. Pederneiras. 2012. Moraceae in Lista de Espécies da Flora do Brasil. Jardim Botânico do Rio de Janeiro. <http:// floradobrasil.jbrj.gov.br/2012/FB010110>. (Acesso em 15/12/2012).

Sá, C.F.C. 2012. Nyctaginaceae in Lista de Espécies da Flora do Brasil. Jardim Botânico do Rio de Janeiro. <http://floradobrasil.jbrj.gov.br/2012/ FB010913 >. (Acesso em 15/12/2012).

Sartori, M.S., F. Poggiani \& V.L. Engel. 2002. Regeneração da vegetação arbórea nativa no sub-bosque de um povoamento de Eucalyptus saligna Smith. localizado no Estado de São Paulo. Scientia Forestalis, 62: 86-103.

Scipioni, M.C., S.J. Longhi, M.M. Araújo \& D.J. Reinert. 2009. Regeneração natural de um fragmento da Floresta Estacional Decidual na Reserva Biológica do Ibicuí-Mirim (RS). Floresta, 39: 675-690.

Scherer, A., F. Maraschin-Silva \& L.R.M. Baptista. 2007. Padrões de interações mutualísticas entre espécies arbóreas e aves frugívoras em uma comunidade de Restinga no Parque Estadual de Itapuã, RS, Brasil. Acta Botanica Brasilica, 21: 203-212.

SMA. 2010. Apresentação (Espécies Exóticas Invasoras). Cadernos da Mata Ciliar, 3: 3.

Silva-Luz, C.L. \& J.R. Pirani. 2012. Anacardiaceae in Lista de Espécies da Flora do Brasil. Jardim Botânico do Rio de Janeiro. <http:// floradobrasil.jbrj.gov.br/2012/FB004411>. (Acesso em 15/12/2012).

Silva, M.C.N.A. \& M.J.N. Rodal. 2009. Padrões das síndromes de dispersão de plantas em áreas com diferentes graus de pluviosidade, $\mathrm{PE}$, Brasil. Acta Botanica Brasilica, 23: 1040-1047.

Silva, R.K.S., A.L.P. Feliciano, L.C. Marangon, R.B.A. Lima \& W.B. Santos. 2012. Estrutura e síndromes de dispersão de espécies arbóreas em um trecho de mata ciliar, Sirinhaém, Pernambuco, Brasil. Pesquisa Florestal Brasileira, 32(69): 1-11.

Silva, W.C., L.C. Marangon, R.L.C. Ferreira, A.L.P. Feliciano \& R.F. Costa-Jr. 2007. Estudo da regeneração natural de espécies arbóreas em fragmento de Floresta Ombró- 
fila Densa, Mata das Galinhas, no Município de Catende, Zona da Mata Sul de Pernambuco. Ciência Florestal, 17: 321-331.

Stevenson, P.R., M.C. Castellanos, J.C. Pizarro \& M. Garavito. 2002. Effects of Seed Dispersal by Three Ateline Monkey Species on Seed Germination at Tinigua National Park, Colombia. International Journal of Primatology, 23: 1187-1204.
Suhs, R.B. \& J.C. Budke. 2011. Spatial distribution, association patterns and richness of tree species in a seasonal forest from the Serra Geral formation, southern Brazil. Acta Botanica Brasilica, 25: 605-617.

Zenni, R.D. \& S.R. Ziller. 2011. An Overview of Invasive Plants in Brazil. Revista Brasileira de Botânica, 34: 431-446.

Recebido em 04.X.2013

Aceito em 10.II.2014 